\title{
Stepwise evolution of a butterfly supergene via duplication and inversion
}

Kang-Wook Kim¹, Rishi De-Kayne*1, Ian J. Gordon ${ }^{2}$, Kennedy Saitoti Omufwoko ${ }^{3}$, Dino J. Martins $^{3,4}$, Richard ffrench-Constant ${ }^{5}$ \& Simon H. Martin ${ }^{1}$

${ }^{1}$ Institute of Evolutionary Biology, University of Edinburgh, Edinburgh, United Kingdom ${ }^{2}$ Centre of Excellence in Biodiversity and Natural Resource Management, RN1, Huye Campus, Huye, Rwanda

${ }^{3}$ Department of Ecology and Evolutionary Biology, Princeton University, Princeton, USA ${ }^{4}$ Mpala Research Centre, Nanyuki, Kenya

${ }^{5}$ Centre for Ecology and Conservation, University of Exeter, Penryn Campus, Penryn, United Kingdom

* these authors contributed equally 


\section{ABSTRACT}

Supergenes maintain adaptive clusters of alleles in the face of genetic mixing. Although usually attributed to inversions, there are few cases in which the specific mechanisms of recombination suppression, and their timing, have been reconstructed in detail. We investigated the origin of the $\mathrm{BC}$ supergene, which controls variation in warning colouration in the African Monarch butterfly, Danaus chrysippus. By generating chromosome-scale assemblies for all three alleles, we identified multiple structural differences. Most strikingly, we find that a region of $>1$ million bp underwent several segmental duplications at least 7.5 million years ago. The resulting duplicated fragments appear to have triggered four inversions in surrounding parts of the chromosome, resulting in stepwise growth of the region of suppressed recombination.

Phylogenies for the inversions are incongruent with the species tree, and suggest that structural polymorphisms have persisted for at least 4.1 million years. In addition to the role of duplications in triggering inversions, our results suggest a previously undescribed mechanism of recombination suppression through independent losses of divergent duplicated tracts. Overall, our findings challenge the idea of instantaneous supergene evolution through a single inversion event, instead pointing towards a stepwise process involving a variety of structural changes.

\section{INTRODUCTION}

Supergenes are clusters of loci at which adaptive combinations of alleles are co-inherited. This can facilitate the maintenance of complex phenotypes under balancing selection [1,2], or link locally adapted alleles in the face of migration [3]. Supergenes are commonly associated with chromosomal inversion polymorphisms [4], but there are numerous cases in which supergenelike architecture is observed but the precise mechanism of recombination suppression is unknown [5] and/or untested [6,7]. Importantly, a single inversion can only suppress crossing over between derived and ancestral gene orders [8], and therefore cannot explain the existence of supergenes with more than two alleles. These require more complex mechanisms, such as a series of nearby or overlapping inversions $[9,10]$. Other forms of genomic rearrangement that disturb local synteny may also contribute to recombination suppression [11], but there are few well-characterised cases. When multiple structural changes are involved, autosomal supergenes may arise through a stepwise process [12] similar to the progressive spread of recombination suppression in sex chromsome evolution [13]. To test this idea, it is necessary to determine both the mechanisms of recombination suppression and their timing.

To investigate the steps involved in supergene evolution, we studied the BC locus in Danaus chrysippus. This dispersive butterfly is known as the African Monarch, African Queen or Plain Tiger in different parts of its broad range. It has bright warning colouration indicating its toxicity [14], and is divided into distinct geographic morphs with partly overlapping ranges [15]. Despite its vast range, genetic differentiation is almost non-existent between distant populations: $F_{\mathrm{ST}}$ is approximately zero across the genome, with the exception of a few broad peaks, including two that are associated with colour differences [7]. This implies selection for the maintenance of local morphs, possibly driven by learned predator avoidance favouring the most common local morph [16]. Colour variation in the forewing is controlled by the $\mathrm{BC}$ supergene on chromosome 
$15[7,17]$. This broad region of suppressed recombination includes two colour patterning loci: B controls brown vs orange variation and localises at the melanin pathway gene yellow, and $\mathrm{C}$ controls the presence/absence of a black forewing tip and white band. Three divergent BC alleles, which determine three distinct forewing phenotypes, have been described to date [7]. A previous genome assembly of one of the alleles (namely 'klugii') revealed that several genes on chromosome 15 had increased in copy number in $D$. chrysippus relative to the outgroup Danaus plexippus (the Monarch), suggesting that gene duplications may have been involved in the evolution of the $\mathrm{BC}$ supergene [7].

To reconstruct the steps in the evolution of the BC supergene, we generated haplotype-resolved chromosomal assemblies for all three of the divergent alleles using long-read sequencing and trio-binning. We traced the evolution of the various structural changes across the phylogeny to determine the sequence of events. Our findings show that the supergene has evolved through multiple steps over the course of several million years, and was preceded by extensive segmental duplications, which likely triggered recombination suppression.

\section{RESULTS}

\section{Haploid assemblies of the three BC alleles}

We generated four new haploid genome assemblies from two F1 individuals by binning reads according to their parent of origin [18] (see Table S1 for accession numbers). In All four assemblies, chromosome 15 (chr15) is represented by 1-4 contigs that could be ordered and oriented by eye (Figure S1). To identify the supergene allele represented by each assembly, we used an 'ancestry painting' approach based on sequence similarity to representative individuals of known genotype [7]. This confirmed that we had assembled all three alleles, as follows: MB18102MAT = 'chrysippus', MB18102PAT = 'klugii' and SB211PAT = 'orientis' (Figure S2). Unexpectedly, the fourth assembly (SB211MAT) showed mixed similarity to all three alleles, suggesting that it may be a rare recombinant that has not yet been observed in the homozygous state (Figure S2, S3).

Using the same ancestry painting procedure, we also confirmed that a previous assembly (Dchry2.2, [19]) represents a 'pure' klugii allele (Figure S2), despite having been assembled from diploid reads from a suspected heterozygous individual. We therefore examined the discarded haplotypic contigs from the Dchry2 assembly and identified the other haplotype, which represents the chrysippus allele (Figure S2). Therefore, in total, we have generated six assemblies of chr15: two representing the klugii allele, two representing the chrysippus allele, one representing the orientis allele, and one representing a putative recombinant. The two independent assemblies of the klugii and chrysippus alleles allowed us to check for assembly errors. In both cases the independent assemblies of the same chr15 allele were co-linear with no obvious errors detected (Figure S4).

\section{Multiple structural rearrangements in the evolution of the $\mathrm{BC}$ supergene}

We predicted that each of the three BC alleles has undergone independent structural changes resulting in recombination suppression. We therefore first analysed the alignments between 
each allele and the outgroup $D$. plexippus assembly, assumed to be structurally unaltered from the ancestral state. As predicted, each allele shows multiple structural differences from $D$. plexippus (Figure 1, Figure S1). Most of these differences are unique to one of the three BC alleles, implying that they are derived changes. The structural events involve four adjacent chromosomal regions. The most strikingly rearranged is Region 3 (1.3 megabases [Mb]), which has been fragmented and duplicated multiple times in all three resulting alleles. Many of the duplicated fragments have been inverted and translocated. These multiple duplications result in chr15 being considerably longer in $D$. chrysippus than in D. plexippus, with striking variation in length also seen among the BC supergene alleles due to each carrying a different complement of duplicated fragments of Region 3 (Figure 1). Region three shows elevated levels of transposable elements (TEs), specifically LINEs, in both the D. plexippus and $D$. chrysippus (Figure S5), suggesting that TE activity may have triggered the segmental duplications.

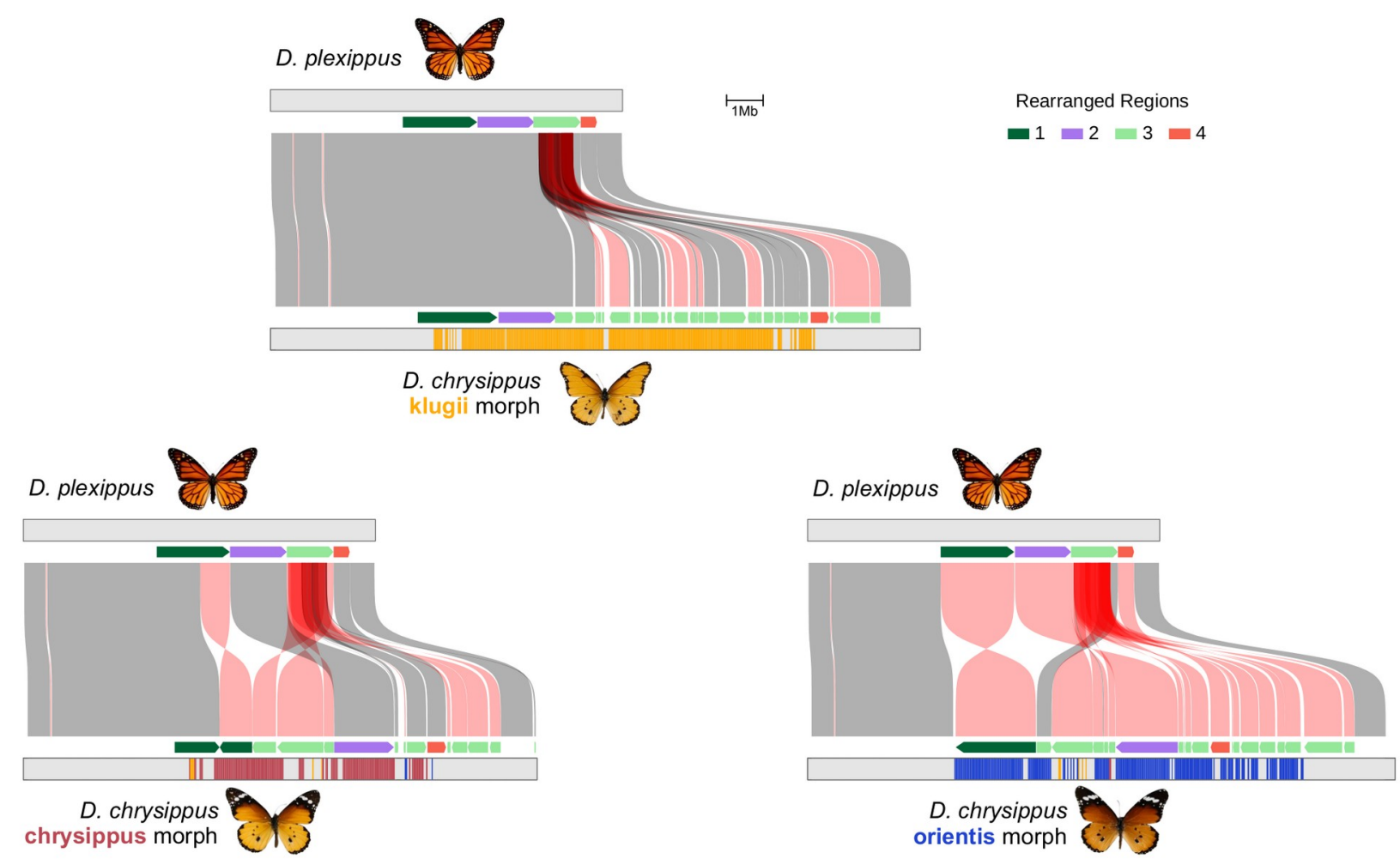

Figure 1. Structural changes in D. chrysippus supergene alleles relative to the outgroup. Connecting boxes indicate syntenic blocks (see Methods for details) between the outgroup ( $D$. plexippus, MEX_DaPlex assembly) and each of the three BC supergene alleles in D. chrysippus. Blocks that are syntenic but in the reverse orientation are coloured red. Partial transparency is used to reveal duplicated syntenic blocks. Coloured arrows indicate the four regions that we identified as having experienced independent rearrangements in $D$. chrysippus, with the direction of the arrow indicating their relative orientation. The coloured bars indicate 'ancestry painting' in $50 \mathrm{~kb}$ windows along the $D$. chrysippus chromosomes (see also Figure S2). Regions lacking coloured bars could not be assigned ancestry because they showed high sequence similarity to two or more different morphs. 
Although duplications can cause assembly errors if their sequences are too similar, this issue does not appear to have affected our assemblies. Independent assemblies of the same allele show strong similarity (Figure S4), and read depth analyses show no evidence of large collapsed repeats (Figure S6). This suggests that the duplications and repeats are distinct enough to assemble separately, which is also confirmed by the lack of highly similar repeats showing up whole genome alignments (Figure S4).

The other three Regions - 1 (2 Mb), 2 (1.5 Mb) and 4 (0.4 Mb) - are each inverted in the orientis allele (Figure 1). A portion of Region 1 is also independently inverted in the chrysippus allele, but appears to share the same right-hand inversion breakpoint. In both the orientis and chrysippus alleles Region 2 is shifted to the right due to upstream insertion of duplicated fragments of Region 3. In all of these simpler rearrangements, either one or both edges are bordered by a duplicated fragment of Region 3. We therefore hypothesised that the duplications of Region 3 may have occurred first and triggered subsequent inversions.

\section{Multiple duplications deep in the history of the Danaus genus}

To test the hypothesis that multiple duplications of Region 3 initiated the formation of the supergene, we estimated the copy number of each gene on chr15 in six other Danaus species and an outgroup using short read data (see Table S1 for accession numbers). Most of the 476 genes for which we had sufficient data to quantify copy number were present as a single copy in all species. However, a cluster of 10 genes in Region 3 shows high copy numbers in all three $D$. chrysippus morphs as well as three additional species: Danaus petilia (Australia), Danaus gilippus (Americas), and Danaus melanippus (Asia) (Figure S7, Table S4). Our inferred species tree, based on 5954 gene alignments confirms that the multiple duplications of Region 3 have a single origin at the base of subgenus anosia (Figure $2 \mathrm{~B}$ ). The tree also resolves previous uncertainty regarding the placement of $D$. eresimus, which groups with $D$. plexippus and $D$. erippus, all of which lack the multiple duplications of Region 3 genes. 


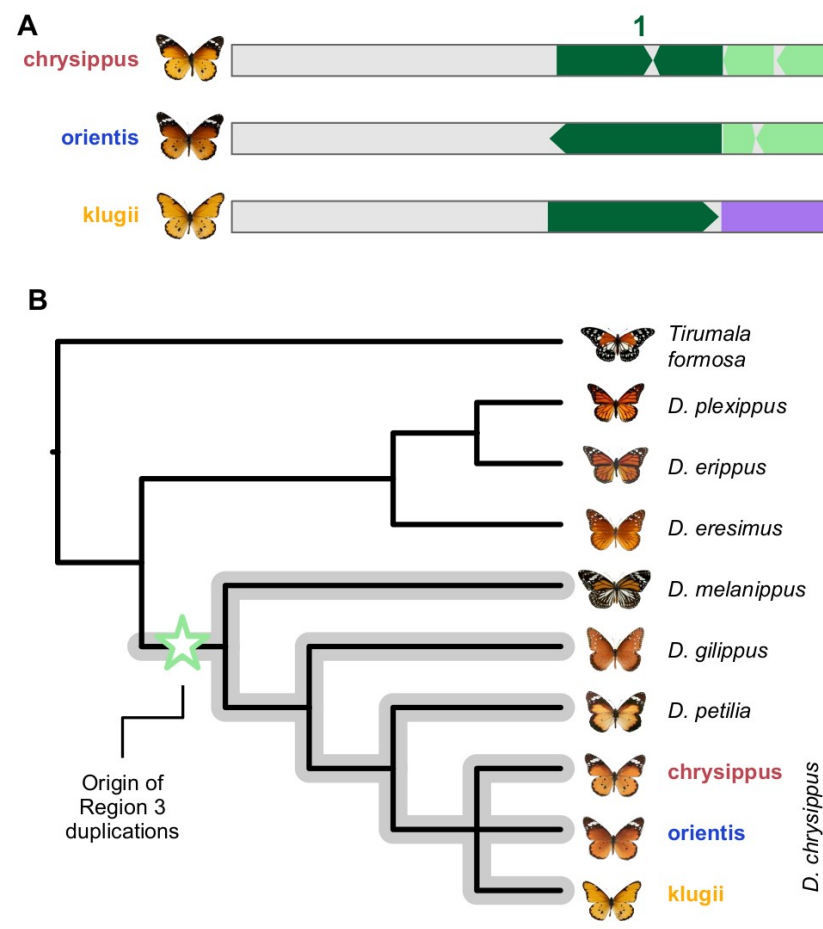

C

24

$\stackrel{1 \mathrm{Mb}}{\longmapsto}$

D

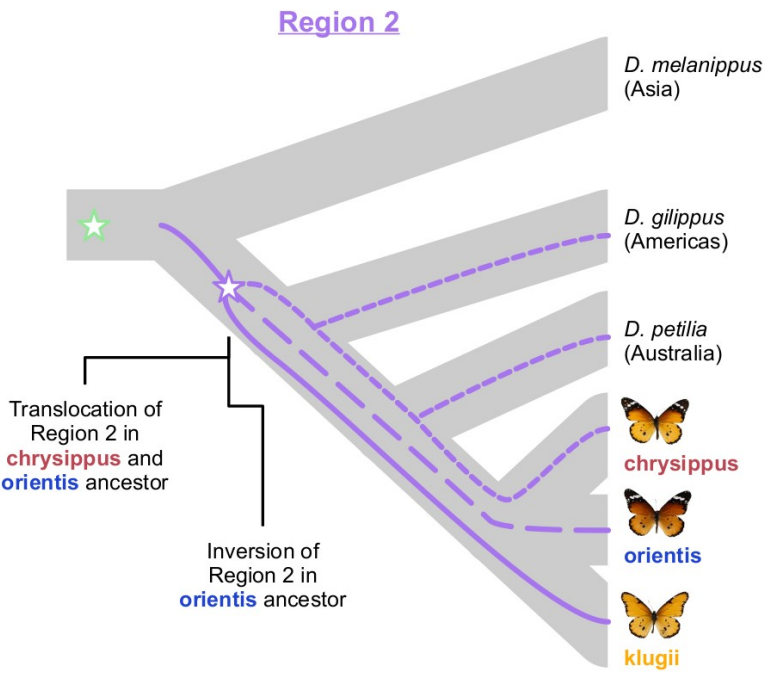

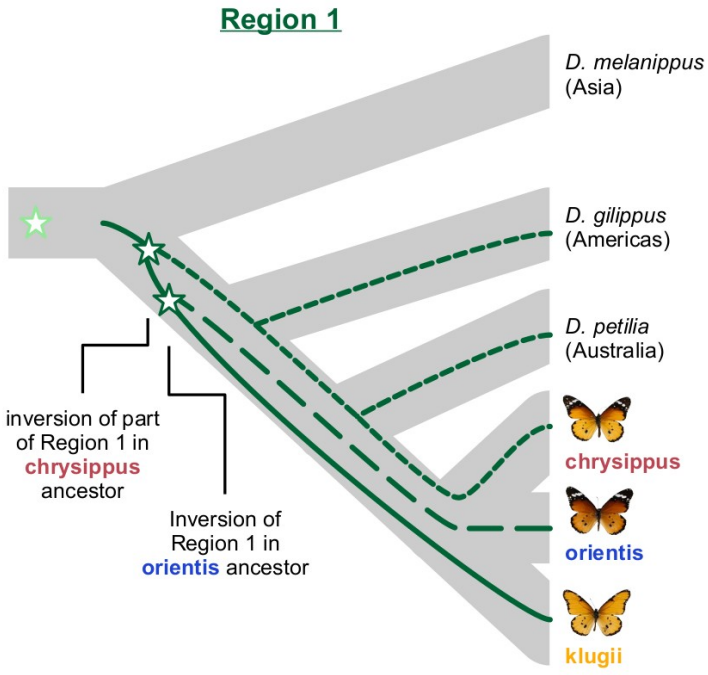

E

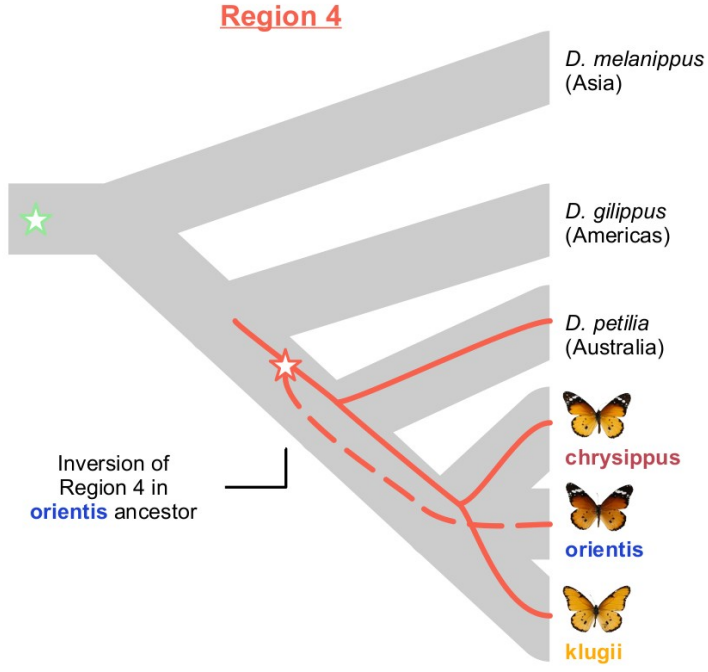

Figure 2. Tracing structural changes across the Danaus phylogeny. A. The structure of the three supergene alleles, showing the position and orientation of Regions 1, 2 and 4, which are interspersed by fragments of Region 3. B. Species tree for Danaus inferred using ASTRAL, based on 5954 gene alignments. The grey clade indicates all species with elevated gene copy number in Region 3 (Figure S7, Table S4). The green star indicates the inferred origin of the Region 3 duplications. C, D, and E. Inferred genealogies for regions 1, 2 and 4 (see Figure S6 for full maximum-likelihood trees). All three genealogies are incongruent with the species branching order. Node ages are therefore positioned to show the most likely origins of the structural variants (indicated by stars) to allow for the observed incongruence.

We estimated a lower bound for the origin of the copy number variation at the base of the anosia clade. $d_{\mathrm{a}}$ (absolute sequence divergence adjusted for estimated divergence in the ancestral species) at putatively-neutral 4-fold degenerate sites between $D$. chrysippus and $D$. 
melanippus is $8.5 \%(95 \% \mathrm{Cl}=8.4-8.6)$. Although we cannot convert this directly to a split time in years as the mutation rates and generation times for this clade are unknown, we can calibrate our estimate according to the estimated split between Danaus and Tirumala of 12.7 million years ago (Chazot et al. 2019). $d_{\mathrm{a}}$ at 4-fold degenerate sites between $D$. chrysippus and $T$. formosa is $14.5 \%(95 \% \mathrm{Cl}=14.3,14.6)$. This gives an estimate of 7.5 million years ago (MYA) as a lower bound for the origin of the Region 3 duplications.

There is consistent variation in estimated copy number for each Region 3 gene among species (Figure 2, Table S4), with the highest copy numbers observed in the D. chrysippus morphs. This is most parsimoniously explained by further duplications in the $D$. chrysippus lineage, but there is also evidence for loss of copies (see below). Further work will be required to determine whether all of the duplicated copies are expressed and have phenotypic consequences.

\section{Structural polymorphisms persist through two speciation events}

We next investigated the history of the three simpler rearrangements on chr15 by generating maximum likelihood trees from concatenated coding sequences within each of the three regions involved (Figure S8). The trees for all of the inversions are incongruent with the species branching order (Figure 3C-E), implying that the inversion polymorphisms are ancient and have persisted through speciation events. In Region 1, the first event appears to have been an inversion of part of the region in the ancestor of the chrysippus allele, followed by a separate inversion of the whole of Region 1 in the ancestor of the orientis allele (Figure 3C). Both $D$. gilippus and $D$. petilia are most closely related to the chrysippus allele. This may reflect a scenario in which both $D$. gilippus (found in the Americas) and $D$. petilia (found in Australia) originated through dispersal from North Africa/Asia, where the chrysippus allele is currently found [7]. Using the dating procedure described above, we estimate the split giving rise to $D$. gilippus to 4.1 MYA, implying that the polymorphisms have persisted for at least this long.

Region 2 has also undergone two structural events: an inversion specific to the orientis allele, and a shift (or translocation) caused by insertion of duplicated fragments of Region 3 (Figure 2A). The tree for Region 2 is similar to that for Region 1, indicating recombination suppression among all three supergene alleles pre-dates the emergence of $D$. gilippus and $D$. petilia, who both inherited the chrysippus-like allele (Figure 2D). The lack of resolution in the node separating the three variants suggests that the inversion and translocation happened close in time. Finally, the tree for Region 4 suggests that the inversion specific to the orientis allele occurred after the speciation event that gave rise to $D$. gilippus, but before the emergence of $D$. petilia (estimated at 1.7 MYA), which inherited the ancestral state (Figure 2E).

\section{Sequence divergence reveals the landscape of recombination suppression}

To understand how the different chr15 rearrangements contribute to recombination suppression in the BC supergene, we examined $d_{X Y}$ (absolute sequence divergence between morphs) and $d_{a}$ (divergence corrected for within-population diversity) across the chromosome. Elevated sequence divergence is a more reliable indicator of deep coalescence than relative measures such as $F_{\mathrm{ST}}$ [20], and is therefore a useful metric to identify regions of the genome at which recombination has been suppressed between segregating alleles for a long time. For each pair 
of alleles, clear regions of elevated sequence divergence are detectable, corresponding approximately with the regions that are rearranged between the pair (Figure S9). In Regions 1 and 2 , the level of sequence divergence is very high $\left(d_{X Y} \sim 6 \%, d_{a} \sim 4 \%\right)$, consistent with these rearrangements having occurred several million years ago, whereas Region 4 shows notably lower divergence, consistent with a more recent inversion (Figure S9). In all three alleles - most notably klugii - some of the duplicated fragments of Region 3 have little or no sequence homology with the other alleles (Figure S9). This implies that some of the paralogs are too divergent to allow cross-mapping of short reads, likely reflecting ancient duplications followed by independent losses of copies in each allele (see Discussion).

\section{DISCUSSION}

By generating chromosome-scale assemblies for all three known alleles of the D. chrysippus BC supergene we have uncovered a stepwise process of supergene formation, with a pivotal early role of segmental duplications. Our results suggest two ways in which duplications may contribute to recombination suppression. The first has been described previously: duplicated regions can trigger inversions by promoting ectopic crossovers [21,22]. Meiotic pairing between paralogs can create inverted loops that lead to inversions if crossovers occur on both sides (Figure 5A). In the BC supergene, two of the inverted regions (2 and 4) are bordered on both sides by duplicated fragments, consistent with their involvement in the inversions. Another two inversions involving Region 1 are bordered only on one side by duplicated fragments. The reuse of breakpoints in multiple structural events has been seen elsewhere, and is consistent with a role of repeats in driving recurrent inversion mutations [12].

A

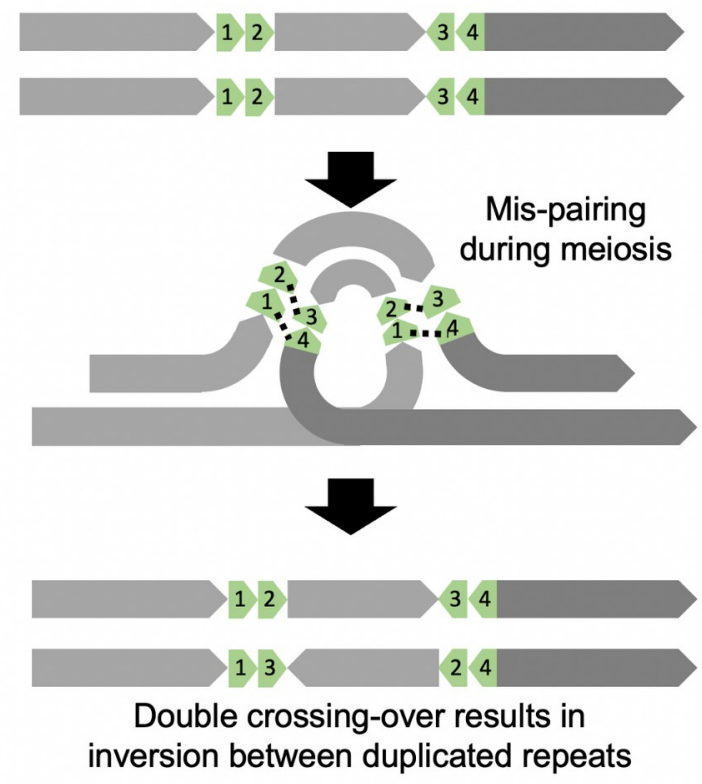

B

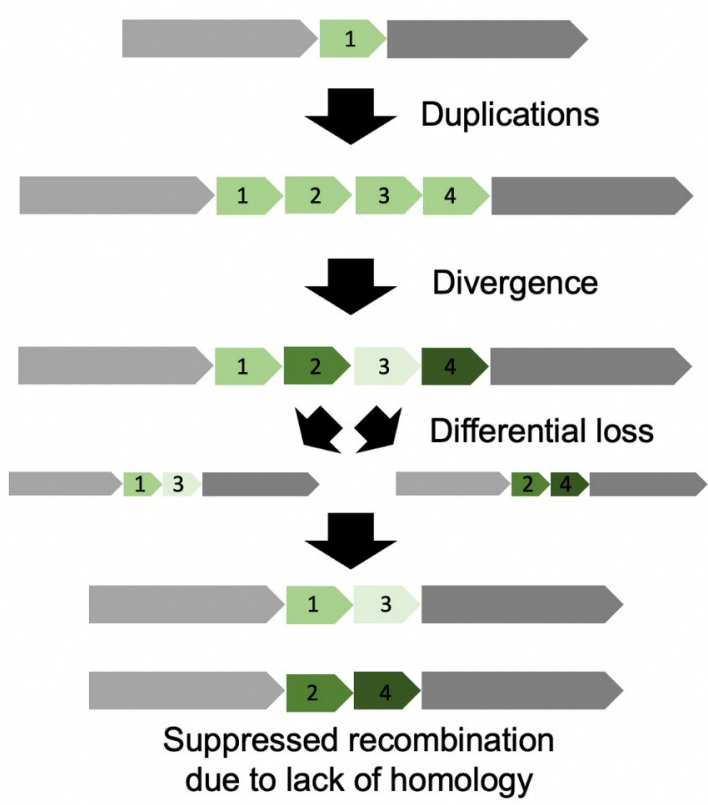

Figure 3. Two mechanisms by which segmental duplications can lead to recombination suppression. A. Ectopic recombination between mis-paired paralogs in different parts of the chromosome can cause an inversion of the intervening region. This mechanism is most relevant when the paralogs are 'young' and still share strong sequence similarity. B. Our proposed model of duplicaplication, divergence and 
differential loss. Over long periods of time, old paralogs may become highly diverged in the sequences. If differential loss of paralogs occurs in different individuals, the remaining copies may be too divergent to pair up and cross over during meiosis.

The second mechanism by which duplications can contribute to recombination suppression has, to our knowledge, not been previously proposed. Our model has three steps (Figure 5B). First, one or more tandem duplications occur and spread to fixation in the population. Second, over a long period, the paralogs become highly diverged through accumulation of mutational differences. Third, independent losses of different paralogs in different individuals leads to the coexistence of haplotypes in a single species that may fail to pair up and form crossovers during meiosis due to lack of homology (Figure S9A). Our model could help to explain the existence of non-recombining regions in the absence of inversions, such as the doublesex polymorphism in Papilio memnon [5]. It remains to be seen whether duplications and copy number variation are a common feature of supergenes in other taxa.

Following the ancient duplications, four independent inversions occurred in a stepwise manner to produce the three divergent BC supergene alleles we see today. This stepwise increase in the range of recombination suppression is similar to processes thought to underlie sexchromosome evolution $[13,23]$. It is possible that the expansion of the BC supergene has allowed the accumulation of additional co-adapted alleles, and potentially extended its adaptive role to traits beyond colour pattern. The modular nature of the inversions also means that recombination can occur between them without creating unbalanced gametes, and thereby generate recombinant supergene alleles. Indeed, wild individuals frequently show mosaic patterns of ancestry across the BC region [7], and one of the four new assemblies described here appears to be a recombinant (Figure S2). These findings, taken together with recent evidence for extensive gene conversion in inversions in Drosophila [24], suggest that the evolution of supergene alleles may be a more dynamic process than previously thought. As long-range sequencing and chromosomal assembly becomes feasible for an increasing number of study systems, we expect that the stepwise mechanisms of recombination suppression described here will prove to be common.

\section{METHODS}

\section{Samples, processing, and sequencing}

To produce haploid assemblies for the three BC alleles, we used a trio binning approach in which long sequencing reads from a diploid sample are binned according to their parent of origin before assembling. We generated two broods (MB181 and SB211) using parents with distinct colour patterns to produce heterozygous F1s carrying two distinct supergene alleles. Specifically, Brood MB181 comprised a father suspected to be homozygous for the klugii allele and a mother suspected to be heterozygous chrysippus/orientis. Brood SB211 comprised a father suspected to be homozygous for the orientis allele and a mother suspected to be heterozygous chrysippus/orientis. From brood MB181, DNA was extracted from a single pupa (MB18102) using the Qiagen MagAttract HMW DNA Kit. Pacific Biosciences (PacBio) sequencing was then carried out using 4 SMRT cells on a PacBio Sequel to produce a total of 34.7 gigabases $(\mathrm{Gb})$ of sequence data. From brood SB211, DNA was extracted from three 
pupae (SB21101, SB21102, SB21104) and sequenced in a single SMRT cell of a PacBio Sequel II to produce $\mathrm{HiFi}$ reads totalling $12.5 \mathrm{~Gb}$ of sequence.

In addition to the long-read sequencing of F1s, we performed Illumina sequencing of parents of both broods for trio binning (but used an aunt for MB181 because the mother was lost). We also generated Illumina reads for the F1 pupa of brood MB181 for polishing the assembly, and from an individual of Tirumala formosa, to serve as an outgroup. DNA extraction was performed using the Qiagen DNEasy Blood and Tissue KIT. Sequencing was performed using the Illumina NovaSeq 600 platform with 300 cycles and an insert size of 350 bp (see Table S1 for sample information and sequencing coverage).

\section{Genome assembly and annotation}

Although trio binning of long reads requires both parents, we reasoned that a sister of the mother could be used to correctly bin reads from certain chromosomes. Lepidopteran females do not undergo crossing over, so daughters share identical chromosomal haplotypes with maternal aunts for some chromosomes. However, we needed to first confirm that the daughter and aunt shared a first-degree relationship for chr15, the chromosome of interest. To this end, we aligned the Illumina reads to a chromosomal-level $D$. plexippus assembly ('Dplex_v4', GCA_009731565, [25]) using BWA v0.7.15 mem [26], converted to BAM format using SAMtools v0.1.18 [27], and split by chromosome using BamTools split v2.5.1 [28]. We applied IBSrelate [29] to each chromosome separately and confirmed that the F1 and aunt had parent-offspring level relationships for around half the chromosomes, including chr15 (Figure S8).

We used Canu v2.1 [30] to perform trio-binning and assembly for MB18102 (Table S2), with estimated genome size of $360 \mathrm{Mb}$ and minimum read length of $2 \mathrm{~kb}$. For brood SB211, we had PacBio HiFi sequence data from three offspring (SB21101, SB21102, SB21104). We therefore first binned reads using the -haplotype option of Canu, and then pooled the binned reads representing each parent to obtain sufficient read depth for both assemblies. For the assembly, both HiFiasm and HiCanu [31] were tested. Based on contiguity, we chose to proceed with the HiFiasm assembly for the paternal reads (SB211PAT), and the HiCanu assembly for the maternal reads (SB211MAT).

We assessed assembly completeness using BUSCO v3.1.0 [32] with arthropoda_odb9 and insecta_odb9 dataset. As expected, biased binning occured in the MB181 and more PacBio reads were assigned to father. The paternal assembly (MB18102PAT) therefore shows a high level of duplication whereas the maternal assembly (MB18102MAT) shows a high level of missing BUSCOs (Table S3). However, we assumed that these issues would only affect the chromosomes that do not show parent-offspring relationships with the aunt, and therefore not affect chr15. For all assemblies, redundant (haplotypic) contigs were removed with Purge Haplotigs [33].

Polishing to correct sequencing errors was performed for the two MB18102 assemblies (the SB211 assemblies did not require polishing as they were generated using PacBio HiFi reads). Two rounds of polishing were carried out simultaneously on the MB18102MAT and MB18102PAT assemblies using Illumina data from the assembled F1 individual in the program Hapo-G (allowing the correct mapping of reads to the correct haplotype). Following polishing, each of the MB18102 and SB211 assemblies were repeat masked and annotated using 
RepeatMasker [34] and BRAKER2 [35] pipeline, respectively (as in [19]; using the same Dchry2.2 repeat library and $D$. plexippus protein sets). The transposable element content of the genome was calculated in $50 \mathrm{~kb}$ windows and the RepeatMasker output using a custom script (Get.TE.Bed.sh; https://github.com/RishiDeKayne/GetTEBed).

\section{Assembly alignment and rearrangement detection}

To identify contigs corresponding to chromosome 15, we performed whole-genome alignment to two chromosomal-level D. plexippus assemblies: 'Dplex_v4' (GCA_009731565, [25]) and 'MEX_DaPlex' (GCA_018135715, [36]). Note that chromosome numbers in the D_plex4 assembly are different, and chr15 corresponds to chr7. For alignment, we used both minimap2 [37] with the 'asm20' parameter preset and the nucmer command in MUMmer v.4.0.0 (Marçais et al. 2018). Alignments were explored visually using the online tool at https://dot.sandbox.bio. For final visualisation, we used the minimap2 alignments to the outgroup $D$. plexippus 'MEX_DaPlex' assembly, and discarded alignments $<500$ bp in length. For comparison among assemblies of $D$. chrysippus supergene alleles, we used minimap2 with preset 'asm10', and discarded alignments of $<2 \mathrm{~kb}$ and with divergence (' $\mathrm{dv}$ value output by minimap2) $<0.2$.

To identify syntenic blocks and delineate rearranged regions, we developed an algorithm that merges adjacent alignments, implemented in the script asynt. $R$ available at https://github.com/simonhmartin/asynt. The algorithm has three steps. First, alignments are split into 'sub-blocks' that each correspond to a unique tract of the reference assembly. Second, subblocks below a minimum size are discarded. Third, adjacent sub-blocks that are in the same orientation and are below some threshold distance apart are merged to yield syntenic blocks. These three steps can be performed iteratively to first identify regions of fine-scale synteny and build these up into larger syntenic blocks. We used three iterations, with minimum sub-block sizes of $200 \mathrm{bp}, 2 \mathrm{~kb}$ and $20 \mathrm{~kb}$, respectively, and a maximum distance between sub-blocks of $100 \mathrm{~kb}$.

\section{Ancestry painting based on sequence divergence}

We compared each assembled chr15 haplotype to wild individuals identified to be homozygous for one of the three supergene alleles in a previous study [7] (see Table S1 for accession numbers). Short reads were aligned using BWA mem v0.7.17 [26] with default parameters. BAM files were generated using SAMtools [27] v1.9 and sorted using PicardTools

(https://broadinstitute.github.io/picard/) SortSam v2.21.1. Duplicate reads were identified and removed using PicardTools MarkDuplicates. Genotypes were called using the repeat-masked references with bcftools v1.10.2 [27,38], using the mpileup, call and filter tools to retain only genotypes with an individual read depth (DP) $\geq 8$ and genotype quality $(G Q) \geq 20$. Diploid genotypes for each individual were exported, along with the haploid assembly genotype using the script parseVCF.py and diversity $(\pi)$ and divergence $\left(d_{X Y}\right)$ between each reference panel and the assembly were computed using the script popgenWindows.py (https://github.com/simonhmartin/genomics_general). Non-overlapping windows of 25,000 genotyped sites were used, and for each reference panel, at least 10,000 sites had to be genotyped in at least one of the individuals. We then calculated $d_{a}$ by subtracting $\pi$ for a given reference panel from $d_{X Y}$. 'Ancestry painting' was performed by assigning, for each window, the 
reference panel that was most similar to the assembly according to $d_{a}$, with the added requirement that the difference between the lowest and second lowest $d_{a}$ value had to be $\geqslant 0.01$ (Figure S2).

\section{Copy number variation}

We estimated gene copy number variation based on read depth of Illumina reads. We used $D$. plexippus (Dplex_v4) as a reference as his species was inferred to represent the ancestral state in gene copy number. Reads were aligned and BAM files were processed as described above. To ensure that copy numbers were comparable among species and not biased by poor read mapping in highly divergent regions, we only considered read depth at exonic sites that had at least one read mapped in all individuals from all species. To this end, we generated a vcf file as described above, but without filtering for depth or genotype quality, and used bcftools view to retain only sites at which all individuals had non-zero depth. Finally, median depth was computed for each gene using the scripts parseVCF.py and windowStats.py (https://github.com/simonhmartin/genomics_general). Genes with $<100$ sites across all exons present in the data set were excluded. Gene copy numbers were estimated by normalising median depths by the median depth across all genes in the chromosome and rounding to the nearest whole number.

\section{Phylogenetic analyses}

We generated alignments for the coding regions of each gene using available short-read Illumina sequence data for seven Danaus species and an outgroup from the sister genus Tirumala that was sequenced for this study (Table S1). The same VCF files described above for copy number analysis were used, with all genotypes with a genotype quality (GQ) $<20$ and read depth (DP) < 5 set to missing data. We then removed all sites with any missing data, converted diploid genotypes to single bases with heterozygous genotypes expressed as IUPAC ambiguity characters, and finally extracted alignments for each gene using the scripts parseVCF.py, filterGeno.py and extractCDSalignments.py available at https://github.com/simonhmartin/genomics_general. After generating these alignments for each gene, we discarded genes where alignments had $>500 \mathrm{Ns}$ and comprised $\geqslant 75 \% \mathrm{Ns}$.

For species tree inference, we further excluded all genes on chromosomes thought to carry rearrangements [7]: chr4 (chr11 in Dplex_v4), chr7 (chr15), chr15 (chr7), chr17 (chr16), chr22 (chr20), chr30 (chr28). The resulting 5954 gene alignments were analysed using the multispecies coalescent tool ASTRAL [39], implemented in the package ParGenes v.1.1.2 ([40]; which produces individual gene trees with RAxML [41] and then initiates an ASTRAL run on this full set of trees).

To explore the history of individual rearrangements we subsetted our filtered set of gene alignments to only the genes found within the bounds of the rearranged region (Region 1 was divided into two to account for the separate inversions in orientis and chrysippus). We then ran RAxML v.8.2.12 [41] on a concatenated alignment for each subset. 


\section{Sequence divergence in sliding windows}

We calculated sequence divergence between reference panels representing each supergene allele using the same genotype data described in the ancestry painting section above. Nonoverlapping windows of 25,000 genotyped sites were used, and $\geq 10,000$ sites had to be genotyped in at least one of the individuals from each panel. Nucleotide diversity $(\boldsymbol{\pi})$ and divergence $\left(d_{X Y}\right)$ were computed using the script popgenWindows.py

(https://github.com/simonhmartin/genomics_general), and we then calculated $d_{a}$ by subtracting the average $\pi$ for a given pair from $d_{X Y}$.

\section{DATA ACCESSIBILITY}

All four assemblies and corresponding annotation are available at the European Nucleotide Archive project accession: XXXXX. Additional data files are provided at http://datadryad.org/XXX, including: repeat library, alignment files, window-based divergence, repeat-content and read depth. Scripts for assembly polishing, the analysis of repeat content, genome annotation, and phylogenetic tree construction are available at https://github.com/RishiDeKayne/Danaus_supergene_structure. Scripts for genome alignment and synteny block inference, ancestry painting and divergence analyses, and read depth and copy number analyses are available at https://github.com/simonhmartin/Danaus_supergene_structure.

\section{REFERENCES}

1. Küpper $C$ et al. 2016 A supergene determines highly divergent male reproductive morphs in the ruff. Nat. Genet. 48, 79-83.

2. Mérot C, Llaurens V, Normandeau E, Bernatchez L, Wellenreuther M. 2020 Balancing selection via life-history trade-offs maintains an inversion polymorphism in a seaweed fly. Nat. Commun. 11, 670.

3. Kirkpatrick M, Barton N. 2006 Chromosome inversions, local adaptation and speciation. Genetics 173, 419-434.

4. Wellenreuther M, Bernatchez L. 2018 Eco-Evolutionary Genomics of Chromosomal Inversions. Trends Ecol. Evol. 33, 427-440.

5. lijima T, Kajitani R, Komata S, Lin C-P, Sota T, Itoh T, Fujiwara H. 2018 Parallel evolution of Batesian mimicry supergene in two Papilio butterflies, P. polytes and $\mathrm{P}$. memnon. Sci Adv 4, eaao5416.

6. Todesco $\mathrm{M}$ et al. 2020 Massive haplotypes underlie ecotypic differentiation in sunflowers. Nature 584, 602-607.

7. Martin SH et al. 2020 Whole-chromosome hitchhiking driven by a male-killing endosymbiont. PLoS Biol. 18, e3000610.

8. Sturtevant AH, Beadle GW. 1936 The Relations of Inversions in the X Chromosome of Drosophila Melanogaster to Crossing over and Disjunction. Genetics 21, 554-604.

9. Jay P, Chouteau M, Whibley A, Bastide H, Parrinello H, Llaurens V, Joron M. 2021 
Mutation load at a mimicry supergene sheds new light on the evolution of inversion polymorphisms. Nat. Genet. 53, 288-293.

10. Joron $\mathrm{M}$ et al. $2006 \mathrm{~A}$ conserved supergene locus controls colour pattern diversity in Heliconius butterflies. PLoS Biol. 4, e303.

11. Ozias-Akins P, Akiyama Y, Hanna WW. 2003 Molecular characterization of the genomic region linked with apomixis in Pennisetum/Cenchrus. Funct. Integr. Genomics 3, 94-104.

12. Yan Z et al. 2020 Evolution of a supergene that regulates a trans-species social polymorphism. Nat Ecol Evol 4, 240-249.

13. Charlesworth D, Charlesworth B, Marais G. 2005 Steps in the evolution of heteromorphic sex chromosomes. Heredity 95, 118-128.

14. Brower LP, Edmunds M, Moffitt CM. 1975 Cardenolide content and palatability of a population of Danaus chrysippus butterflies from West Africa. Journal of Entomology Series A, General Entomology 49, 183-196.

15. Smith DAS, Owen DF, Gordon IJ, Lowis NK. 1997 The butterfly Danaus chrysippus (L.) in East Africa: polymorphism and morph-ratio clines within a complex, extensive and dynamic hybrid zone. Zool. J. Linn. Soc. 120, 51-78.

16. Smith DAS. 1979 The significance of beak marks on the wings of an aposematic, distasteful and polymorphic butterfly. Nature 281, 215-216.

17. Smith DAS, Gordon IJ, Allen JA. 2010 Reinforcement in hybrids among once isolated semispecies ofDanaus chrysippus(L.) and evidence for sex chromosome evolution. Ecol. Entomol. 35, 77-89.

18. Koren S et al. 2018 De novo assembly of haplotype-resolved genomes with trio binning. Nat. Biotechnol. 36, 1174-1182.

19. Singh KS, De-Kayne R, Omufwoko KS, Martins D, Bass C, ffrench-Constant R, Martin SH. 2021 Genome assembly of Danaus chrysippus and comparison with the Monarch Danaus plexippus. Biorxiv BIORXIV/2021/470194.

20. Cruickshank TE, Hahn MW. 2014 Reanalysis suggests that genomic islands of speciation are due to reduced diversity, not reduced gene flow. Mol. Ecol. 23, 3133-3157.

21. Montgomery EA, Huang SM, Langley CH, Judd BH. 1991 Chromosome rearrangement by ectopic recombination in Drosophila melanogaster: genome structure and evolution. Genetics 129, 1085-1098.

22. Arguello JR, Connallon T. 2011 Gene duplication and ectopic gene conversion in Drosophila. Genes 2, 131-151.

23. Otto SP et al. 2011 About PAR: the distinct evolutionary dynamics of the pseudoautosomal region. Trends Genet. 27, 358-367.

24. Korunes KL, Noor MAF. 2019 Pervasive gene conversion in chromosomal inversion heterozygotes. Mol. Ecol. 28, 1302-1315. 
25. Gu L, Reilly PF, Lewis JJ, Reed RD, Andolfatto P, Walters JR. 2019 Dichotomy of Dosage Compensation along the Neo Z Chromosome of the Monarch Butterfly. Curr. Biol. 29, 4071-4077.e3.

26. Li H, Durbin R. 2010 Fast and accurate long-read alignment with BurrowsWheeler transform. Bioinformatics 26, 589-595.

27. Li H et al. 2009 The Sequence Alignment/Map format and SAMtools.

Bioinformatics 25, 2078-2079.

28. Barnett DW, Garrison EK, Quinlan AR, Strömberg MP, Marth GT. 2011

BamTools: a C++ API and toolkit for analyzing and managing BAM files. Bioinformatics 27, 1691-1692.

29. Waples RK, Albrechtsen A, Moltke I. 2019 Allele frequency-free inference of close familial relationships from genotypes or low-depth sequencing data. Mol. Ecol. 28, 35-48.

30. Koren S, Walenz BP, Berlin K, Miller JR, Bergman NH, Phillippy AM. 2017 Canu: scalable and accurate long-read assembly via adaptive k-mer weighting and repeat separation. Genome Res. 27, 722-736.

31. Nurk S et al. 2020 HiCanu: accurate assembly of segmental duplications, satellites, and allelic variants from high-fidelity long reads. Genome Res.

(doi:10.1101/gr.263566.120)

32. Simão FA, Waterhouse RM, loannidis P, Kriventseva EV, Zdobnov EM. 2015 BUSCO: assessing genome assembly and annotation completeness with single-copy orthologs. Bioinformatics 31, 3210-3212.

33. Roach MJ, Schmidt SA, Borneman AR. 2018 Purge Haplotigs: allelic contig reassignment for third-gen diploid genome assemblies. BMC Bioinformatics 19, 460.

34. Smit AFA, Hubley R, Green P. 2015 RepeatMasker Open-4.0. 2013--2015.

35. Hoff KJ, Lomsadze A, Stanke M, Borodovsky M. 2018 BRAKER2: incorporating protein homology information into gene prediction with GeneMark-EP and AUGUSTUS.

Plant and Animal Genomes XXVI

36. Ranz JM et al. 2021 A de novo transcriptional atlas in Danaus plexippus reveals variability in dosage compensation across tissues. Commun Biol 4, 791.

37. Li H. 2018 Minimap2: pairwise alignment for nucleotide sequences.

Bioinformatics 34, 3094-3100.

38. Danecek P et al. 2021 Twelve years of SAMtools and BCFtools. Gigascience 10, giab008 (doi:10.1093/gigascience/giab008)

39. Mirarab S, Reaz R, Bayzid MS, Zimmermann T, Swenson MS, Warnow T. 2014 ASTRAL: genome-scale coalescent-based species tree estimation. Bioinformatics $\mathbf{3 0}$, i541-8.

40. Morel B, Kozlov AM, Stamatakis A. 2019 ParGenes: a tool for massively parallel 
model selection and phylogenetic tree inference on thousands of genes. Bioinformatics 35 , $1771-1773$.

41. Stamatakis A. 2014 RAxML version 8: a tool for phylogenetic analysis and postanalysis of large phylogenies. Bioinformatics 30, 1312-1313.

\section{ACKNOWLEDGEMENTS}

We thank Alex Mackintosh for providing advice on genome assembly and annotation and valuable feedback on our interpretations of the results. We thank Chay Graham for helpful comments on the manuscript. This work was supported by a Royal Society University Research Fellowship (URFIR1\180682) and Enhancement Award (RGFIEAI181071) awarded to SHM, a Swiss National Science Foundation Early Postdoc Mobility Fellowship (P2BEP3_195567) awarded to RDK, and a National Geographic Society Research Grant (WW-138R-17) awarded to IJG. 